\title{
New Physics at Polarized Hadronic Colliders
}

\author{
J.-M. Virey ${ }^{\mathrm{a} *}$ and P. Taxil ${ }^{\mathrm{a}}$ \\ ${ }^{a}$ Centre de Physique Théorique (UPR 7061), CNRS-Luminy, Case 907, F-13288 Marseille Cedex 9, \\ France \\ and \\ Université de Provence, Marseille, France
}

Concerning new physics beyond the Standard Model we explore the discovery and analysis potentials of polarized (hadronic) experiments and we compare with the unpolarized case. For discovery, beam polarization is helpful in the case of purely hadronic new interactions. In any case, beam polarization provides us a unique piece of information on the chiral and flavour structures.

\section{Introduction}

At the Brookhaven National Laboratory it is expected, during the year 2001, to run the Relativistic Heavy Ion Collider (RHIC) in the polarized $\vec{p} \vec{p}$ mode. Actually, this run will be done at an energy $\sqrt{s}=200 \mathrm{GeV}$ and with a luminosity of a few $10^{30} \mathrm{~cm}^{-2} \mathrm{~s}^{-1}$. The nominal energy $\sqrt{\mathrm{s}}=$ $500 \mathrm{GeV}$ and luminosity $\mathcal{L}=2.10^{32} \mathrm{~cm}^{-2} \mathrm{~s}^{-1}$ should be reached in the early months of 2003 , allowing an exposure of $800 p b^{-1}$ in four months running.

The physics potential of the RHIC-Spin experiments has been extensively covered in a recent review paper [1]. In view of the apparent feasability of accelerating polarized protons at very high energies, several other projects are now considered. Indeed, since 1997 several workshops have been organised on the physics interests of the HERA machine with a beam of polarized protons [2. More recently, the future of the LHC has been considered by a working group at CERN. It appears that a polarized option for the proton beams is not excluded. In this workshop, Albert de Roeck reports on such a future polarized LHC option [3]. Note that the physics with polarized beams at hadronic supercolliders has been

\footnotetext{
*Preprint CPT-01/P.4243. To appear in the proceedings of the ECT* workshop "The Spin Structure of the Proton and Polarized Collider Physics", July 2001, Trento, Italy.
}

already explored some time ago [4, 5$]$.

The physics program of the RHIC and HERA polarized experiments is directly connected to the analysis of the QCD structure of the proton. For instance, at RHIC the first part of the program will include precise measurements of the polarization of the gluons, quarks and sea-antiquarks in a polarized proton. This will be done thanks to well-known Standard Model (SM) processes : direct photon, $W$ and $Z$ production, Drell-Yan pair production, heavy-flavor production and the production of jets. The helicity structure of perturbative QCD will be thoroughly tested at the same time with the help of Parity Conserving double spin asymmetries.

However, here we ask an other question: "Concerning New Physics, which kind of information can be obtained from such polarized facilities?"

Of course, the answer is far from trivial. It depends on the various properties of the machine under consideration, like the energy scale, luminosity, type of incident particles, type of process which is analysed ... Nevertheless, several definite advantages of the polarized experiments can be pointed out simply by comparison with the existing results of unpolarized experiments.

In the following we will consider some possible 
manifestations of new physics models which are as most as possible model independent. In particular we consider the presence of Contact Interactions (CI) which mimic the existence of new subconstituants or new interactions. We also consider the presence of new gauge bosons (e.g. $Z^{\prime}$, $W^{\prime}$ or Leptoquarks). In this case one can get a resonant behaviour if the energy is sufficiently high. Conversely we do not consider for the moment the SUSY phenomenology which is certainly very rich but highly model dependent.

Presently, the strongest constraints on such new physics models are obtained from LEP, HERA and Tevatron colliders. In particular, for processes involving leptons, either in the initial state like at LEP and HERA, or in the final state like the Drell-Yan process at Tevatron, some strong and complementary constraints have been obtained on new physics models. For instance, the characteristic energy scale $\Lambda$ of $\mathrm{CI}$ is constrained to be above $4 \mathrm{TeV}$ for leptonlepton or lepton-quark CI [6]. The mass of a $Z^{\prime}$ which is present in popular GUT models (extra $U(1)$ 's or Left-Right models) should be above $600-700 \mathrm{GeV}$ [6]. The precise values of the bounds depend on the details of the models for each collider.

If polarization of the beams is present, we do not expect any improvements on these constraints. Specific examples have been considered in [7] for $e q$-CI and in [8] for Leptoquarks, in the context of HERA. It was shown that the polarization of the beams is useless to reveal some new physics effects, but it is very useful to identify the origin of the new interaction, if the latter is discovered first by the unpolarized experiments.

Conversely, when we consider some pure hadronic processes the situation is drastically different. Indeed, in a context like the one of the Tevatron, it appears that the analysis of hadronic processes, like jet or $b \bar{b}$ productions, is far to be easy. In such channels the systematic errors, coming both from the experimental and the theoretical sides, are rather large. For instance, for jets the CDF and D0 collaborations are still in disagreement [9.10]. For $b \bar{b}$ production, the compar- ison between data and theory is still problematic [11]. Therefore, the bounds on new physics models from such hadronic channels are quite low in comparison to the ones from leptonic processes. For example, the last published analysis of D0 on $q q$-CI gives a limit of $\Lambda>2.2 \mathrm{TeV}$ (from jet production) [9], a factor two below the constraints obtained on $e q$-CI from the same experiment (from the Drell-Yan process).

In this case, the availability of polarized proton beams can be very helpful, especially in the hope of a discovery. Indeed, the main difference between an unpolarized and a polarized experiment concerns the basic observables used in the analysis of data : we shift from unpolarized cross sections to spin asymmetries. The spin asymmetries have the net advantage to minimize systematic errors in comparison to cross sections [1]. This is especially true for channels with huge systematics like pure hadronic processes. For example, it has been shown that the RHIC Spin experiment is competitive with the Tevatron for the search of a $q q$-CI 12] or of a leptophobic $Z^{\prime}$ [13], in spite of an energy four times lower !

To summarize we can say that polarized beams allow to define a new type of observables : spin asymmetries.

Firstly, for processes with high systematic errors, like pure hadronic channels, the use of spin asymmetries reduce strongly these systematics and allow the polarized experiment to have a high discovery potential. In the next section we examplify this result with the analysis of the effects of $q q$-CI and leptophobic $Z^{\prime}$ at RHIC (and at a polarized LHC in section 3).

On the other hand, the analysis of spin asymmetries yields a unique opportunity to get some informations on the chiral structure and also on the flavour structure of the new interaction. Some examples in the context of HERA can be found in [7.87. In the third section we show the results of an analysis done in the context of the LHC with polarized protons, to pin down the origin of a new generic $Z^{\prime}$ boson from the Drell-Yan process [14].

\section{The RHIC case (jet production)}




\subsection{Observable and models}

At RHIC, running in the $\vec{p} \vec{p}$ mode, it will be possible to measure with a great precision the single Parity Violating (PV) asymmetry $A_{L}$ :

$A_{L}=\frac{d \sigma_{(-)}-d \sigma_{(+)}}{d \sigma_{(-)}+d \sigma_{(+)}}$

where only one of the proton is polarized (the sign \pm refer to the helicity of the polarized proton). The cross section $d \sigma_{(\lambda)}$ means the one-jet production cross section in a given helicity configuration, $p_{1}^{(\lambda)} p_{2} \rightarrow j e t+X$, estimated at $\sqrt{s}=500 \mathrm{GeV}$ for a given jet transverse energy $E_{T}$, integrated over a pseudorapidity interval $\Delta \eta=1$ centered at $\eta=0$.

The new physics models that can be tested at RHIC with jet production are the followings :

- First 12 one can think to a simple phenomenological contact interaction which could represent the consequences of quark compositeness. Such (color singlet and isoscalar) terms are usually parametrized following Eichten et al. [15] :

$\mathcal{L}_{q q}=\epsilon \frac{\pi}{2 \Lambda^{2}} \bar{\Psi} \gamma_{\mu}\left(1-\eta \gamma_{5}\right) \Psi \cdot \bar{\Psi} \gamma^{\mu}\left(1-\eta \gamma_{5}\right) \Psi$

where $\Psi$ is a quark doublet, $\epsilon$ is a sign, $\eta$ can take the values \pm 1 or 0 and $\Lambda$ is the compositeness scale. In the following we will consider the $L L^{-}$case with Left-handed chiralities $(\eta=1)$ and constructive interference with QCD amplitudes which corresponds to $\epsilon=-1$.

- Second, we can consider some new neutral gauge bosons with general Left and Right-handed couplings to each given quark flavor $q$ :

$\mathcal{L}_{Z^{\prime}}=\kappa \frac{g}{2 \cos \theta_{W}} Z^{\prime \mu} \bar{q} \gamma_{\mu}\left[C_{L}^{q}\left(1-\gamma_{5}\right)+C_{R}^{q}\left(1+\gamma_{5}\right)\right] q(3)$

the parameter $\kappa=g_{Z^{\prime}} / g_{Z}$ being of order one. A particular class of models, called leptophobic $Z^{\prime}$, is poorly constrained by present data since they evade the constraints coming from leptonic channels. Such models appear in several string-inspired scenarios 16. Non supersymmetric models can also be constructed 17. Here, for simplicity, we consider only the model $A$ of [13] and we refer the reader to this reference [13] for more details. In addition, it was advocated in 18 that such a boson could appear with a mass close to the electroweak scale and a mixing angle to the standard $Z$ close to zero.

\subsection{Results}

We give in Table 1 the $95 \%$ C.L. limits on $\Lambda \equiv \Lambda_{L L^{-}}$(eq.2) one gets [19], at lowest order, from a comparison between the SM asymmetry $A_{L}$ and the Non-Standard one. A $\chi^{2}$ analysis is used.

\begin{tabular}{ccccc}
\hline$L\left(\mathrm{fb}^{-1}\right)$ & 0.8 & 4 & 20 & 100 \\
$\Lambda(\mathrm{TeV})$ & 3.2 & 4.55 & 6.15 & 7.55 \\
\hline
\end{tabular}

Table 1 : Limits on $\Lambda_{L L^{-}}$at $95 \%$ CL for RHIC with $\sqrt{s}=500 \mathrm{GeV}$.

One can compare the bounds at $\sqrt{s}=500 \mathrm{GeV}$ and $L=0.8 \mathrm{fb}^{-1}$ with the ones after some luminosity upgrades : $L=0.8 \mathrm{fb}^{-1}$ is the nominal luminosity expected after 4 months of run. $L=20 \mathrm{fb}^{-1}$ may be reached in the future with the same amount of running time but after an upgrade of the machine [20]. $4 \mathrm{fb}^{-1}\left(100 \mathrm{fb}^{-1}\right)$ represents $5 \times 4$ months running with the designed (future) nominal luminosity.

This table can be compared with the last published analysis of the D0 experiments at Tevatron [9]: $\Lambda>2.2 \mathrm{TeV}$ (95\% C.L.) from the dijet mass cross section. From these figures we have extrapolated a limit at Tevatron of $3.2 \mathrm{TeV}(3.7 \mathrm{TeV})$ with a $1 \mathrm{fb}^{-1}\left(10 \mathrm{fb}^{-1}\right)$ exposure. If we compare these numbers with the ones from Table 1, it is clear that RHIC has a better sensitivity than Tevatron, if sufficient data are accumulated.

Turning now to the case of a leptophobic $Z^{\prime}$, we present in Fig.1 the constraints on the parameter space $\left(\kappa, M_{Z^{\prime}}\right)$ obtained [19] from $A_{L}$ in the flipped SU(5) model (model $A$ of [13]). The dotted curves correspond to $\sqrt{s}=500 \mathrm{GeV}$ and the dashed curves to $\sqrt{s}=650 \mathrm{GeV}$. From bottom to top they correspond to an integrated luminosity $L=1,10,100 \mathrm{fb}^{-1}$. It appears that the increase in luminosity is more efficient than the increase 
in energy. Therefore the high luminosity scenario has to be supported even if the RHIC $p p$ c.m. energy remains at its "low" value.

We display also in Fig. 1 the inferred constraints coming from the published results of UA2 21, CDF [10] and D0 [22] experiments. The form of the forbidden areas result from a combination of statistical and systematic errors. For high $M_{Z^{\prime}}$ one is looking for some unexpected high- $E_{T}$ jet events and the main uncertainty is statistical in nature. For instance, the upper part of the "CDF area" is well below the one of D0 because of the well-known excess observed by CDF at high- $E_{T}$. In the future (run II) the increase in statistics will improve the bounds in the $\left(\kappa, M_{Z^{\prime}}\right)$ plane by enlarging the upper part of the CDF and D0 areas (or will lead to a discovery). For relatively low $M_{Z^{\prime}}$ values, the main problem comes from the large systematic errors for "low" $E_{T}$ jets. Due to these systematics, at Tevatron, even with a high statistics it will be difficult to probe the low $\kappa$ region for $M_{Z^{\prime}} \leq 400 \mathrm{GeV}$ or to close the windows around $M_{Z^{\prime}} \simeq 300$ and $100 \mathrm{GeV}$. In this respect, as can be seen from Fig.1, the RHIC-Spin measurements at high luminosity should allow to cover this region and to get definite conclusions, if the new interaction violates parity.

To conclude, we have seen that RHIC is competitive/complementary with the Tevatron to discover a new weak force belonging purely to the quark sector.

\section{The LHC case}

The LHC will give acess to a completely new energy domain. Hence the whole community is expecting to find, at least, some physics beyond the SM. However, besides the problem of discovery, the problem of the identification of the nature of a new interaction is fundamental and much more difficult.

\subsection{Leptonic channels}

The existence of new vector bosons is a common prediction of many scenarios going beyond the SM. The most popular models are based on an expanded gauge symmetry group which contains

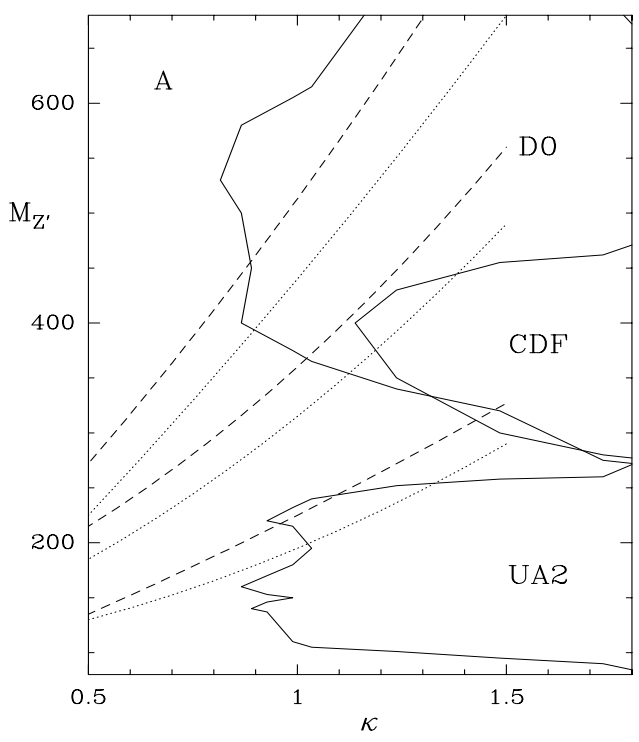

Figure 1. Bounds on the parameter space for leptophobic flipped $\mathrm{SU}(5) Z^{\prime}$ models (see text).

extra $U(1)$ and/or extra $S U(2)$ group(s) leading, after breaking of the symmetry, to the presence of new massive gauge bosons. In general, the masses of these new particles are not fixed by the theory. On the other hand their couplings to ordinary fermions and gauge bosons are determined in each particular model. Here we will not review all the existing models but simply quote the models considered in [14. Namely, we consider the continuous $E_{6}$, Left-Right (LR) and Y models. We have simulated also more exotic models where the couplings to fermions are completely fixed : the "Sequential Standard Model" (SSM), the "BESS" models, and a preonic model labeled by "HYP". Additional models and all references can be found in [14].

Concerning $Z^{\prime}$ production, all the LHC studies have shown that it will not be difficult to produce a massive $Z^{\prime}$ and to detect it in the leptonic 
mode through the Drell-Yan process. The discovery limit is around $M_{Z^{\prime}} \simeq 5 \mathrm{TeV}$ from the analysis of the unpolarized cross section [23]. The sensitivities of the spin asymmetries are well below, roughly of the order of $2 \mathrm{TeV}$ [14]. So, we recover the result that polarized beams are not necessary to discover some new physics effects through leptonic signatures.

Concerning the question of the identification, it has been explored by many authors within unpolarized $p p$ collisions (see e.g. 24] and references therein). The observables at hand are essentially the forward-backward angular asymmetry $A_{F B}$ and some decay widths. It turns out that the observables should allow to pin down some particular models but not to treat some more general cases. Then polarization should help.

Considering that only one proton beam is polarized one can focus on the process $\vec{p}_{a} p_{b} \rightarrow$ $\ell^{+} \ell^{-} X$ in the vicinity of the $Z^{\prime}$ peak. Then, one can define two PV spin-dependent quantities :

$$
\begin{aligned}
& A_{L R}(Y, M)=\frac{d \sigma^{-}-d \sigma^{+}}{d \sigma^{-}+d \sigma^{+}} \\
& A_{F B}^{P o l}(Y, M)=\frac{\left(d \sigma_{F}^{-}-d \sigma_{B}^{-}\right)-\left(d \sigma_{F}^{+}-d \sigma_{B}^{+}\right)}{\left(d \sigma_{F}^{-}+d \sigma_{B}^{-}\right)+\left(d \sigma_{F}^{+}+d \sigma_{B}^{+}\right)}
\end{aligned}
$$

Here $d \sigma^{ \pm}$stands for $d \sigma^{ \pm} / d M, \pm$ corresponding to the helicity of the polarized proton, $M$ being the invariant mass of the lepton pair, and the cross sections are integrated over some rapidity interval $\pm Y$. Note that the measurement of the single spin asymmetry $A_{L R}$ does not require the identification of the charge of the outgoing leptons. $A_{F B}^{P o l}$ is a polarized forward-backward asymmetry which reflects the angular dependence of the subprocess spin asymmetry.

It appears that $A_{L R}$ is independent of the final state couplings (leptonic or whatever) and that $A_{F B}^{P o l}$ goes like the product of initial state and final state couplings (as goes the well-known unpolarized $A_{F B}$ ). This shows the complementarity of these two spin asymmetries.

We present in Figs.2-3 the results of our calculations 14] under the form of combined plots of $A_{F B}$ versus $A_{L R}$ and $A_{F B}^{P o l}$ versus $A_{L R}$, at LHC, for $M_{Z^{\prime}}=1 \mathrm{TeV}$. Note that for $M_{Z^{\prime}}=2 \mathrm{TeV}$ the figures are very similar. The error bars correspond to the statistical error obtained with 1000 $e^{+} e^{-}+1000 \mu^{+} \mu^{-}$events (polarization of the beam is assumed to be $100 \%$ ).

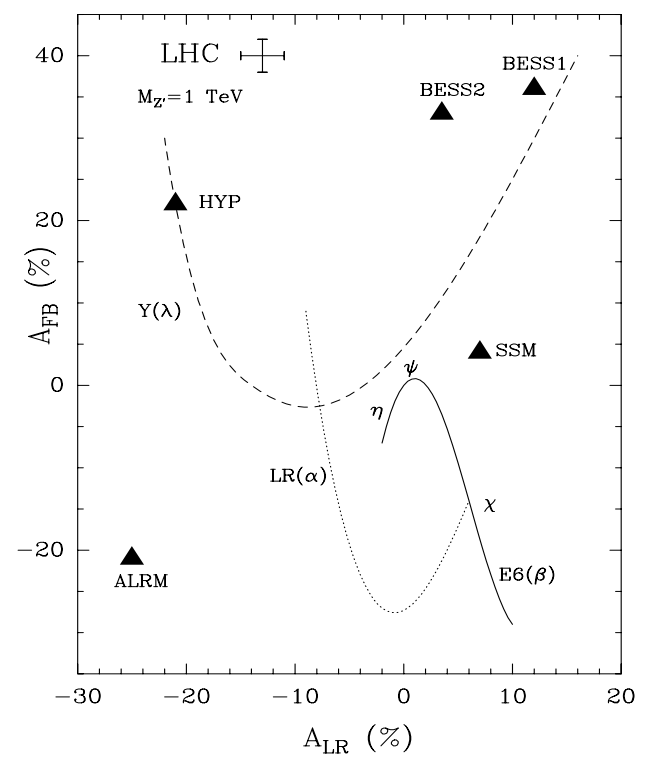

Figure 2. $A_{F B}$ versus $A_{L R}$ according to the various models.

As can be seen from Figs.2-3, it is now very easy to separate the various models, appart from very particular points where some accidental crossing occurs due to the equality of the couplings.

\subsection{Hadronic channels}

The example above shows that beam polarization is useful to analyse some new physics effects but not to discover them. It is a peculiarity of the leptonic channels. For hadronic channels, like jet production we recover similar results as in the RHIC case. Indeed, for $q q$-CI one expect a limit of the order of $\Lambda \simeq 25 \mathrm{TeV}$ from the unpolarized jet cross section with $L=30 \mathrm{fb}^{-1}$ and $\sqrt{s}=14 \mathrm{TeV}$ 


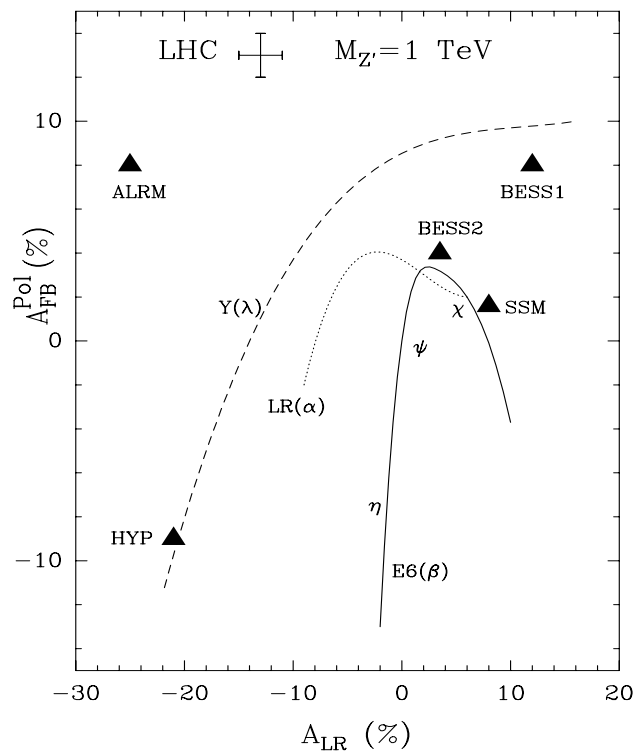

Figure 3. $\quad A_{F B}^{P o l}$ versus $A_{L R}$ according to the various models.

[23]. With the same $L$ and $\sqrt{s}$ values but with polarized protons, we obtain $\Lambda \simeq 50 \mathrm{TeV}$ from $\mathrm{PV}$ spin asymmetries. For leptophobic $Z^{\prime}$ similar results are obtained : the unpolarized jet cross section is sensitive to masses up to $2 \mathrm{TeV}$, but one can reach $3.5 \mathrm{TeV}$ thanks to PV spin asymmetries.

Consequently, we stress again that polarized beams are well suited to discover a new weak force in the hadronic sector.

\section{REFERENCES}

1. G. Bunce, N. Saito, J. Soffer and W. Vogelsang, Ann. Rev. Nucl. Part. Sci. 50 (2000) 525 .

2. "1997 Workshop on Physics with Polarized Protons at HERA", Eds. A. De Roeck and T. Gehrmann; DESY-proceedings-199801; " $3^{\text {rd }}$ UK Phenomenology Workshop on HERA Physics", Durham, UK, September
1998, Eds. R. Devenish et al.; J. Phys. G25, 1269 (1999); "Workshop on Polarized Protons at High Energies - Accelerator Challenges and Physics Opportunities", DESYHamburg, Germany, May 1999, Eds. D. Barber et al.

3. A. De Roeck in these Proceedings.

4. C. Bourrely et al., Phys. Rep. 177 (1989) 319; P. Taxil, Riv. Nuov. Cim 16, n.11 (1993)

5. Polarized Collider Workshop AIP Conference Proceedings 223, Eds. J. Collins et al.

6. Particle Data Group, Eur. Phys. J. C15, 1 (2000).

7. J.-M. Virey, Eur. Phys. J. C8, 283 (1999).

8. P. Taxil, E. Tuğcu and J.-M. Virey, Eur. Phys. J. C14, 165 (2000).

9. B. Abbott et al.(D0 Collaboration), Phys. Rev. D64 (2001) 032003.

10. F. Abe et al., Phys. Rev. D55 (1997) R5263.

11. F. Abe et al. (CDF Collaboration), Phys. Rev. Lett. 75, 264 (2000); B. Abbott et al.(D0 Collaboration), Phys. Rev. Lett. 85, 5068 (2000).

12. P. Taxil and J.-M. Virey, Phys. Lett. B364 (1995) 181.

13. P. Taxil and J.-M. Virey, Phys. Lett. B441 (1998) 376.

14. A. Fiandrino and P. Taxil, Phys.Rev. D44 (1991) 3490, and Phys. Lett.B293 (1992) 242.

15. E. Eichten, K.Lane and M. Peskin, Phys. Rev. Lett. 50, 811 (1983), E. Eichten, et al., Rev. Mod. Phys. 56 (1984) 579.

16. J.L. Lopez and D.V. Nanopoulos, Phys. Rev. D55 (1997) 397; J.D. Lykken in Snowmass 1996, ed. D.G. Cassel, L. Trindle Gennari, R.H. Siemann, p.891; K.S. Babu, C. Kolda and J. March-Russell, Phys. Rev. D54 (1996) 4635 ; D57 (1998) 6788.

17. K. Agashe, M. Graesser, I. Hinchliffe and M. Suzuki, Phys. Lett. B385 (1996) 218; H. Georgi and S.L. Glashow, Phys. Lett. B387, 341 (1996).

18. M. Cvetič et al, Phys. Rev. D56 (1997) 2861.

19. P. Taxil and J.-M. Virey, hep-ph/0109094.

20. N. Saito in "14th International Spin Physics Symposium", Osaka, 2000, K. Hatanaka et al. eds., AIP Conf. Proceedings 570, AIP, New York, 2001. 
21. J. Alitti et al., Zeit. f. Phys. C49 (1991), 17; Nucl. Phys. B400 (1993), 3.

22. B. Abbott et al., FERMILAB-Conf-97/356E.

23. ATLAS Collaboration, Atlas detector and physics performance, Technical Design Report, May 1999.

24. M. Cvetič in "International Europhysics Conference on High Energy Physics", Marseille, 1993. 